\title{
Astragaloside IV prevents kidney injury caused by iatrogenic hyperinsulinemia in a streptozotocin-induced diabetic rat model
}

\author{
KE-QIANG HE ${ }^{1,2}$, WEI-ZU LI ${ }^{1}$, XIAO-QING CHAI ${ }^{2}$, YAN-YAN YIN ${ }^{1}$, YAN JIANG ${ }^{4}$ and WEI-PING LI ${ }^{1,3}$ \\ ${ }^{1}$ Department of Pharmacology, College of Basic Medicine, Anhui Medical University, Hefei, Anhui 230032; \\ ${ }^{2}$ Department of Anesthesiology, Affiliated Provincial Hospital of Anhui Medical University, Hefei, Anhui 230001; \\ ${ }^{3}$ Department of Pharmacology, Anqing Medical and Pharmaceutical College, Anqing, Anhui 246000; \\ ${ }^{4}$ Department of Pathology, Anhui Medical University, Hefei, Anhui 230032, P.R. China
}

Received December 3, 2016; Accepted November 8, 2017

DOI: $10.3892 / \mathrm{ijmm} .2017 .3265$

\begin{abstract}
Diabetic patients are able to manage their blood glucose with exogenous insulin but, ultimately, remain at risk of diabetic nephropathy (DN). Long-term use of insulin may lead to iatrogenic hyperinsulinemia, which has been suggested to cause kidney injury. However, there are no effective interventions for iatrogenic hyperinsulinemia leading to kidney damage. In the present paper, the hypothesis that astragaloside IV (AS-IV), a novel saponin purified from Astragalus membranaceus (Fisch) Bunge, may prevent DN in iatrogenic hyperinsulinemic diabetic rats through antioxidative and anti-inflammatory mechanisms was investigated. Diabetes was induced with streptozotocin (STZ) $(55 \mathrm{mg} / \mathrm{kg})$ by intraperitoneal injection in rats. At 1 week following STZ injection, the diabetic rats were treated with Levemir subcutaneously for 4 weeks. Diabetic rat insulin levels $>30 \mu \mathrm{U} / \mathrm{ml}$ were considered as iatrogenic hyperinsulinemia. Rats were divided into six groups ( $\mathrm{n}=8$ per group): Iatrogenic hyperinsulinemic rats, and iatrogenic hyperinsulinemic rats treated with Tempol and AS-IV at $2.5,5$ and $10 \mathrm{mg} / \mathrm{kg} / \mathrm{day}$, intragastric infusion, for 12 weeks. The normal rats were used as a non-diabetic control group. AS-IV ameliorated albuminuria, mesangial cell proliferation, basement membrane thickening and podocyte foot process effacement in iatrogenic hyperinsulinemic rats. In iatrogenic hyperinsulinemic rat renal tissues, malondialdehyde, interleukin-1 $\beta$ (IL-1 $\beta$ ), tumor necrosis factor- $\alpha$ (TNF- $\alpha)$, type IV collagen and laminin levels were increased, whereas glutathione peroxidase and superoxide dismutase activity levels were decreased. Nicotinamide adenine dinucleotide phosphate oxidase 4 expression and extracellular signal-regulated kinase 1/2 (ERK1/2) activation
\end{abstract}

Correspondence to: Professor Wei-Ping Li, Department of Pharmacology, College of Basic Medicine, Anhui Medical University, 81 Meishan Road, Hefei, Anhui 230032, P.R. China

E-mail: lwp19@126.com

Key words: iatrogenic hyperinsulinemia, diabetic nephropathy, astragaloside IV, oxidative stress, inflammation were upregulated, and canonical transient receptor potential cation channel 6 (TRPC6) protein expression was downregulated. However, all these abnormalities were attenuated by AS-IV. These findings suggested that AS-IV prevented rat kidney injury caused by iatrogenic hyperinsulinemia by inhibiting oxidative stress, IL-1 $\beta$ and TNF- $\alpha$ overproduction, downregulating ERK1/2 activation, and upregulating TRPC6 expression.

\section{Introduction}

Diabetic nephropathy (DN) is the leading cause of end-stage renal disease (ESRD). Currently, there is no effective treatment for DN, and the prevention of the occurrence and progression of DN has become a serious medical challenge. Intensive insulin treatment has been shown in large prospective randomized studies to delay the onset and progression of DN in patients with type 1 and 2 diabetes (1) by lowering blood glucose levels and thus preventing hyperglycemia-associated damage (2). However, the use of insulin may result in iatrogenic hyperinsulinemia (3-6), which can also lead to kidney damage $(4,5)$.

In the kidneys, nicotinamide adenine dinucleotide phosphate (NADPH) oxidase is the predominant source of reactive oxygen species (ROS). NADPH oxidase 4 (Nox4) is the key subunit of NADPH oxidase expressed in mesangial cells, and Nox4-derived ROS are the major contributor to renal morphological changes and functional abnormalities in DN (7). It has been reported that in vitro insulin treatment results in a rapid increase in $\mathrm{H}_{2} \mathrm{O}_{2}$ generation within podocytes, as well as an increase in the surface expression of Nox4 in cultured podocytes (8). High concentrations of insulin promoted pancreatic stellate cell proliferation and extracellular signal-regulated kinase 1/2 (ERK1/2) phosphorylation, which resulted in pancreatic islet fibrosis (9). High concentrations of insulin may also contribute to renal extracellular matrix (ECM) accumulation in diabetes (10). These results suggested that high concentrations of insulin could promote oxidative stress, cell proliferation, and ECM accumulation in vitro. Oxidative stress serves an important role in DN. Regarding the role of ROS in the activation of transforming growth factor- $\beta$ (TGF- $\beta$ ) signalling, it has 
been observed that NADPH oxidase exerts a key role in the TGF- $\beta 1$-mediated activation of kidney myofibroblast and fibronectin extra domain A expression through the Smad3 and ERK signalling pathways (11). NADPH oxidase-mediated renal oxidative stress promotes albuminuria in DN (12). In addition, inhibition of Nox4 oxidase has been demonstrated to reduce whole kidney and glomerular hypertrophy in the diabetic cortex and glomeruli (13), suggesting that Nox4 may have a direct involvement in renal hypertrophy, perhaps via a mechanism involving Nox4-derived, ROS-mediated Akt/PKB and ERK1/2 activation (13). The detrimental role of NADPH oxidase in the development of DN has been further confirmed by a study in which treatment with NADPH oxidase inhibitors markedly attenuated the progression of nephropathy by reducing the occurrence of albuminuria and preventing the development of glomerulosclerosis through a reduction of renal oxidative stress (14). The inflammation pathways also serve central roles in the progression of DN (15). Inflammation increases insulin resistance by activating the ERK pathway (16). However, at the present time, a complete understanding of renal oxidative stress, inflammation and ERK protein expression in the context of iatrogenic hyperinsulinemic diabetic rats, or how to intervene in these processes, has yet to be fully elucidated.

Canonical transient receptor potential cation channels (TRPCs), as members of the transient receptor potential (TRP) superfamily, are $\mathrm{Ca}^{2+}$-permeable cation channels widely expressed in a series of tissues and cells (17). The TRPC family comprises seven members, designated as TRPC1-7 (18). Among these, TRPC6 is closely associated with kidney disease (19). In vitro, the effects of insulin on TRPC6 were mimicked by treating podocytes with $\mathrm{H}_{2} \mathrm{O}_{2}(8)$. However, the effects of intensive insulin therapy on TRPC6 in iatrogenic hyperinsulinemic diabetic rats remain unclear, and the present study was designed in an attempt to address this issue.

Astragaloside IV (AS-IV), a purified small molecular saponin, is one of the main active ingredients of Radix Astragali, which has been reported to possess comprehensive biological properties, including antioxidant, anti-inflammatory, and immunoregulatory effects (20). It has been reported that AS-IV significantly inhibits renal oxidative stress and apoptosis in acute kidney injury rodent models (21). Previous work in our laboratory demonstrated that AS-IV prevents damage to human mesangial cells through inhibition of the NADPH oxidase/ROS/Akt/nuclear factor- $\kappa \mathrm{B}(\mathrm{NF}-\kappa \mathrm{B})$ pathway under high glucose conditions (7). However, the effects of AS-IV on iatrogenic hyperinsulinemic streptozotocin (STZ)-induced diabetic rats under intensive insulin therapy have not yet been elucidated. The purpose of the present study was to examine the hypothesis that AS-IV prevents iatrogenic hyperinsulinemia due to kidney injury in diabetic rats by suppressing oxidative stress and inflammation, and regulating TRPC6 in the field of DN.

\section{Materials and methods}

Animals. The present study was approved by the Animal Care and Use Committee of Anhui Medical University, and all studies were conducted in accordance with guidelines for the Care and Use of Laboratory Animals adopted by the Committee on the Care and Use of Laboratory Animals at Anhui Medical University. Age- and weight-matched male Sprague-Dawley rats weighing 180-200 g were purchased from the Experimental Animal Center of Anhui Medical University (certificate no. SCXK 2014-0007). Rats were housed in individual cages in a temperature-controlled room with a 12/12-h light-dark cycle (starting at 09:00 a.m.) and allowed to acclimatize to the environment for 1 week prior to initiation of the various interventions.

Induction of diabetes mellitus. Freshly prepared STZ (Sigma Chemical Co.; now Merck KGaA, Darmstadt, Germany) was dissolved in $0.01 \mathrm{M}$ citrate buffer ( $\mathrm{pH} 4.5$ ) and administered intraperitoneally to the rats at $55 \mathrm{mg} / \mathrm{kg}$. The age-matched control group received citrate buffer only. On the third or fourth day following STZ administration, blood glucose levels were measured using an ACCU-CHEK performa glucometer (Roche Diagnostics GmbH, Mannheim, Germany) with a drop of blood extracted from the tail vein. Rats with blood glucose levels $>16.7 \mathrm{mmol} / \mathrm{l}$ were considered diabetic (22).

Experimental study design. At 1 week following STZ injection, the diabetic rats were treated with the long-acting insulin analog, Levemir (Novo Nordisk, Bagsvaerd, Denmark) subcutaneously (s.c.) for 4 weeks at $6 \mathrm{U} / \mathrm{day}$. In addition, age-matched control rats (control, $n=8$ ) were treated with saline s.c. for the same period. Subsequently, at 8:00 a.m., 4 weeks after the induction of diabetes, the morning insulin concentration was determined from tail vein samples, obtained under sodium pentobarbital anesthesia from all rats. Insulin levels $>30 \mu \mathrm{U} / \mathrm{ml}$ in the diabetic model rats were considered to represent the condition of hyperinsulinemia (23). These diabetic rats with hyperinsulinemia were randomly divided into the hyperinsulinemic (HINS; $\mathrm{n}=8$ ), the AS-IV-2.5 (i.e., treated with $2.5 \mathrm{mg} / \mathrm{kg}$ AS-IV daily; $\mathrm{n}=8$ ), the AS-IV-5 (treated with $5 \mathrm{mg} / \mathrm{kg}$ AS-IV daily; $\mathrm{n}=8$ ), the AS-IV-10 (treated with $10 \mathrm{mg} / \mathrm{kg}$ AS-IV daily; $n=8)$ and the Tempol $(n=8)$ groups. Diabetic rats in the HINS group continued with s.c. insulin treatment for 12 weeks. The AS-IV-2.5, AS-IV-5 and AS-IV-10 groups also continued to receive s.c. insulin for 12 weeks, but AS-IV $2.5,5$ and $10 \mathrm{mg} / \mathrm{kg} /$ day intragastric (i.g.) administration protocols were also continued for 12 weeks in each corresponding group. Diabetic rats in the Tempol group were given Tempol (Merck KGaA) in their drinking water ( $1 \mathrm{mmol} / \mathrm{l}$ ) for 12 weeks (Tempol group; $\mathrm{n}=8$ ). The control group was treated with saline i.g. over the course of the same time period.

Determination of blood glucose levels and serum levels of glycated hemoglobin (HbAlc) and insulin. At 9:00 a.m., morning blood glucose concentrations were determined at 1 - or 2 to 4-week intervals in all groups using an ACCU-CHEK per forma glucometer (Roche Diagnostics $\mathrm{GmbH}$ ) on blood samples obtained from tail veins. $\mathrm{HbA}_{1 \mathrm{c}}$ and serum insulin levels were determined on blood samples obtained from tail veins under sodium pentobarbital anesthesia at the end of the 12-week study period using a Variant-II analyser (Bio-Rad, Laboratories, Inc., Hercules, 
CA, USA) and a sensitive rat antibody radioimmunoassay (EMD Millipore, Billerica, MA, USA). Subsequent biochemical markers were detected as described below.

Determination of biochemical markers and urinary albumin . Serum creatinine (CREA), blood urea nitrogen (BUN), alanine transaminase (ALT) and aspartate aminotransferase (AST) levels were determined on the blood samples obtained from the abdominal aorta using an Olympus auto analyser (AU640; Olympus, Tokyo, Japan). All rats were kept in individual metabolic cages for $24 \mathrm{~h}$ urine collection at the end of 4,8 and 12 weeks of drug treatment. Urinary albumin concentrations were measured using a turbidimetric inhibition immunoassay kit (Nanjing Jiancheng Bioengineering Institute, Nanjing, China), according to the manufacturer's protocol.

Determination of oxidative stress-related markers, pro-inflammatory cytokines and ECM. After blood sampling, the kidneys were quickly removed from the animals, and the surrounding fat was cleaned. The renal tissue was sheared from the edge of the upper pole of the kidney and homogenized in 9X volume of ice-cold normal saline. Subsequently, the homogenates were centrifuged $(4,000 \mathrm{rev} / \mathrm{min}, 2683 \mathrm{x} \mathrm{g}$ at $4^{\circ} \mathrm{C}$ for $10 \mathrm{~min}$ ). Levels of malondialdehyde (MDA), glutathione peroxidase (GSH-Px), superoxide dismutase activity (SOD), interleukin-1 $\beta$ (IL-1 $\beta$ ), tumor necrosis factor- $\alpha($ TNF- $\alpha)$ and type IV collagen (COl-IV) in the kidney were measured according to the manufacturer's protocol (Nanjing Jiancheng Bioengineering Institute). Levels of laminin (LN) in the kidney were measured according to a different manufacturer's protocol (Nanjing SenBeijia Biological Technology Co., Ltd., Nanjing, China). Levels of IL- $1 \beta$, TNF- $\alpha$, COl-IV and LN were detected using the enzyme-linked immunosorbent assay (ELISA) method. Levels of MDA (thiobarbituric acid method), GSH-Px (colorimetric method), SOD (hydroxylamine method) were also detected using the manufacturer's kits (Nanjing Jiancheng Bioengineering Institute).

Routine histology. Rat kidneys were excised to determine the morphological changes in the kidney. Renal cortical tissue slices were obtained (5- $\mu \mathrm{m}$ thick), and portions were fixed in neutral buffered formalin for routine paraffin embedment and subsequent tissue staining by hematoxylin and eosin (H\&E) and Jones' periodic acid Schiff (PAS) for bright-field microscopic evaluation. The slides were examined under a light microscope at a magnification of $x 400$ by a pathologist blind to the experimental profile. In PAS sections, the purple color in glomeruli was defined as 'positive', and the positive area of each glomerulus was measured using the JD801 morphological microscope image analysis system (JEDA Science-Technology Development Co., Ltd., Jiangsu, China). The positive score of the glomeruli one rat was calculated by averaging positive scores of 30 glomeruli from the same rat (24). Six rats were examined in each group.

Electron microscopy. To determine the structural changes in podocyte morphology, electron microscopic morphometric evaluation was performed using routine protocols. Renal cortex samples were cut into $1 \mathrm{~mm}^{3}$ pieces, incubated on ice, immediately fixed in $2.5 \%$ glutaraldehyde solution, and then embedded. Ultrathin sections were examined under a transmission electron microscope (TEM). The remaining cortex was sliced and frozen by immersion in liquid nitrogen for subsequent detection by western blot analysis, as described below.

Western blot analysis. Kidney cortex was homogenized in lysis buffer on ice with a homogenizer. The supernatants were collected after centrifuging at 10,000 rev./min at $16,770 \mathrm{x}$ g for $15 \mathrm{~min}$ at $4^{\circ} \mathrm{C}$. Protein concentration in the supernatant was measured using the Bradford acid (BCA) protein assay (Pierce; Thermo Fisher Scientific, Inc., Waltham, MA, USA). Equal amounts of protein extracts were fractionated on $10 \%$ sodium dodecyl sulfate-polyacrylamide gel electrophoresis (SDS-PAGE), and then transferred onto polyvinylidene difluoride (PVDF) membranes (EMD Millipore). After having been blocked with 5\% non-fat milk in Tris-buffered saline with Tween-20 (TBST, pH 7.6) for $1 \mathrm{~h}$ at room temperature, the membranes were incubated with the indicated primary antibodies [anti-Nox4 (1:1,000, BS6796), anti-ERK1/2 (1:1,000, BS3628), anti-phospho-ERK1/2 (1:1,000, BS5016) (all from Bioworld Technology, Inc., St. Louis Park, MN, USA); and anti-TRPC6 (1:1,000, ab12249) and anti- $\beta$-actin $(1: 1,000$, ab8227) (both from Abcam, Cambridge, MA, USA)] overnight at $4^{\circ} \mathrm{C}$. The membranes were rinsed three times with TBST and incubated with the respective secondary antibodies (1:10,000 dilutions of each antibody) for $1 \mathrm{~h}$ at room temperature. The protein bands were visualized with SuperSignal ${ }^{\mathrm{TM}}$ West Femto Maximum Sensitivity Substrate (Thermo Fisher Scientific Inc.) and captured using a Bioshine ChemiQ 4600 Mini Chemiluminescence imaging system (Ouxiang Scientific Instrument Co. Ltd., Shanghai, China). The optical density of each band was quantified using ImageJ software (National Institutes of Health, Bethesda, MD, USA) and normalized to the intensity of $\beta$-actin.

Statistical analysis. Data are presented as the means \pm standard deviation. Each experiment was repeated at least three times independently. Statistical analysis was performed using SPSS 16.0 for Windows (SPSS, Inc., Chicago, IL, USA). Statistical differences among groups were analysed by one-way ANOVA. Pairwise comparisons were made using the Bonfferoni test. $\mathrm{P}<0.05$ was considered to indicate a statistically significant difference.

\section{Results}

Effects of AS-IV on blood glucose and serum levels of insulin and $H b A_{I c}$. To determine the effects of AS-IV on blood glucose, serum insulin, and $\mathrm{HbA}_{1 \mathrm{c}}$, all groups except the control group were subjected to intensive insulin treatment (Fig. 1A and B). Regular checks of blood glucose levels demonstrated that diabetic rats had good glycemic control. No statistically significant differences were observed in blood glucose or $\mathrm{HbA}_{1 \mathrm{c}}$ among the six groups $(\mathrm{P}>0.05)$. These results also implied that AS-IV and Tempol did not lead to an alteration in the blood glucose levels. Compared with the control group, STZ-induced diabetic rats exhibited 

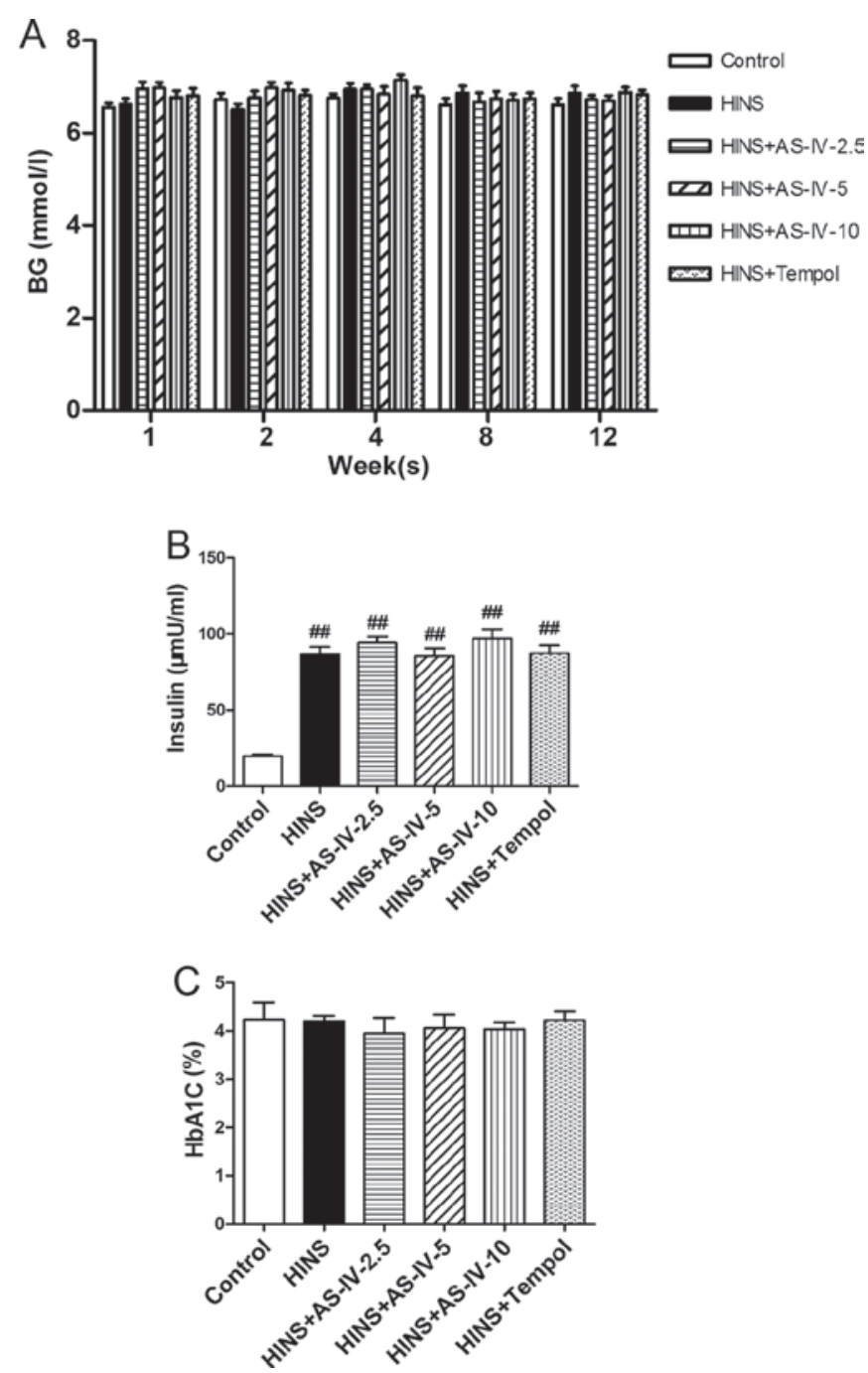

Figure 1. Effects of AS-IV on BG, serum insulin and $\mathrm{HbA}_{1 \mathrm{C}}$ in iatrogenic hyperinsulinemic diabetic rats. (A) BG level of all the groups were examined after 1, 2, 4, 8 and 12 weeks, showing that AS-IV did not affect the levels. (B) Serum insulin level of all groups after 12 weeks. (C) $\mathrm{HbA}_{1 \mathrm{C}}$ level of all groups after 12 weeks, showing that all groups exhibited a good glycemic control. As AS-IV had no impact on the insulin level, the long-term use of insulin may therefore lead to iatrogenic hyperinsulinemia. Results are expressed as the means \pm standard deviation ( $n=8 /$ each group). Refer to the Materials and methods section for details of the assignment of the groups. ${ }^{\# \#} \mathrm{P}<0.01$ vs. control. AS-IV, astragaloside IV; $\mathrm{BG}$, blood glucose; $\mathrm{HbA}_{1 \mathrm{C}}$, glycated hemoglobin; HINS, hyperinsulinemic.

very high insulin levels $(\mathrm{P}<0.05)$ (Fig. 1B). This reveals that the long-term use of insulin may lead to iatrogenic hyperinsulinemia, and that AS-IV and Tempol had no impact on insulin levels.

Effects of AS-IV on serum levels of biochemical markers and urinary albumin. No statistically significant differences in the levels of CREA, BUN, ALT or AST among the six groups were identified ( $\mathrm{P}>0.05)$ (Fig. 2A-D). This suggested that the AS-IV doses used in the experiment did not damage renal or liver function. Compared with the control group, diabetic rats had higher levels of albuminuria $(\mathrm{P}<0.05)$. Treatment with AS-IV significantly ameliorated albuminuria in iatrogenic hyperinsulinemic rats. This protective effect was evident at a dose as low as $5 \mathrm{mg} / \mathrm{kg}$ /day (Fig. 2E). AS-IV reduced albuminuria time-dependently in diabetic rats, which was evident at least 8 weeks after AS-IV treatment. Treatment with AS-IV (at the doses of 5 and $10 \mathrm{mg} / \mathrm{kg} /$ day), or Tempol for 12 weeks, decreased the severity of albuminuria (Fig. 2E). Diabetic rats treated with AS-IV or Tempol were revealed to differ from normal control rats at each time-point. Thus, AS-IV or Tempol treatment was shown to ameliorate iatrogenic hyperinsulinemic kidney damage, although without restoring levels to the baseline. Even in the model group, the urinary albumin excretion rate was far from reaching clinical diagnostic criteria for $\mathrm{DN}$.

Effects of AS-IV on oxidative stress-associated markers. To assess the antioxidant effects of AS-IV, the oxidative stress-associated markers, MDA, GSH-Px and SOD, were measured. Compared with the control group, MDA levels were increased in the HINS and AS-IV-2.5 groups $(\mathrm{P}<0.01)$ (Fig. 3A), whereas GSH-Px and SOD levels were reduced in the HINS, AS-IV-2.5 and AS-IV-5 groups $(\mathrm{P}<0.01)$ (Fig. 3B and $C$ ). These results suggested that marked oxidative stress was occurring in the iatrogenic hyperinsulinemic rats, in addition to their having a reduced antioxidant capacity. However, as shown in Fig. 3A-C, AS-IV significantly reduced the elevation of renal tissue levels of MDA, and increased the elevation of tissue levels of GSH-Px and SOD, effects that were evident at a dose as low as $2.5 \mathrm{mg} / \mathrm{kg} / \mathrm{day}$ for MDA and GSH-Px (but not for SOD; the increase for SOD was observed at a dose of AS-IV as low as $5 \mathrm{mg} / \mathrm{kg} /$ day). These results demonstrated the marked antioxidative capacity of AS-IV. Tempol was also able to reduce MDA, and increase GSH-Px and SOD.

$A S-I V$ decreases the secretion of pro-inflammatory cytokines, and decreases abnormal ECM synthesis. The effects of AS-IV on pro-inflammatory cytokines, such as IL-1 $\beta$ and TNF- $\alpha$, were examined (Fig. 4A and B). The tissue level of IL-1 $\beta$ in the control group was significantly lower compared with that in the HINS, AS-IV-2.5 and AS-IV-5 groups ( $\mathrm{P}<0.01$; Fig. 4A). TNF- $\alpha$ levels in the normal control group were significantly lower compared with those in the other groups, with the exception of the Tempol group $(\mathrm{P}<0.01$; Fig. 4B). Increases in the levels of pro-inflammatory cytokines are able to promote ECM accumulation and to mediate tissue injury. However, AS-IV significantly reduced the renal tissue levels of IL- $1 \beta$ and TNF- $\alpha$, which was evident at a dose as low as $2.5 \mathrm{mg} / \mathrm{kg} / \mathrm{day}$ for TNF- $\alpha$ Fig. 4B) and $5 \mathrm{mg} / \mathrm{kg} /$ day for IL-1 $\beta$ (Fig. 4A). Tissue levels of Col-IV and LN in the normal control group were identified to be significantly lower compared with those in the HINS group ( $\mathrm{P}<0.01)$ (Fig. 4C and D). Increases in the levels of Col-IV and LN are indicative of an increase in the synthesis of renal ECM. AS-IV significantly reduced renal tissue levels of Col-IV and LN, which was evident at a dose as low as $5 \mathrm{mg} / \mathrm{kg} /$ day. Thus, AS-IV may attenuate basement membrane thickening in iatrogenic hyperinsulinemic rats. Tempol was also able to reduce renal tissue levels of IL-1 $\beta$, TNF- $\alpha$, Col-IV and LN.

AS-IV alleviates the histopathological alterations of kidneys in iatrogenic hyperinsulinemic rats. At 12 weeks 

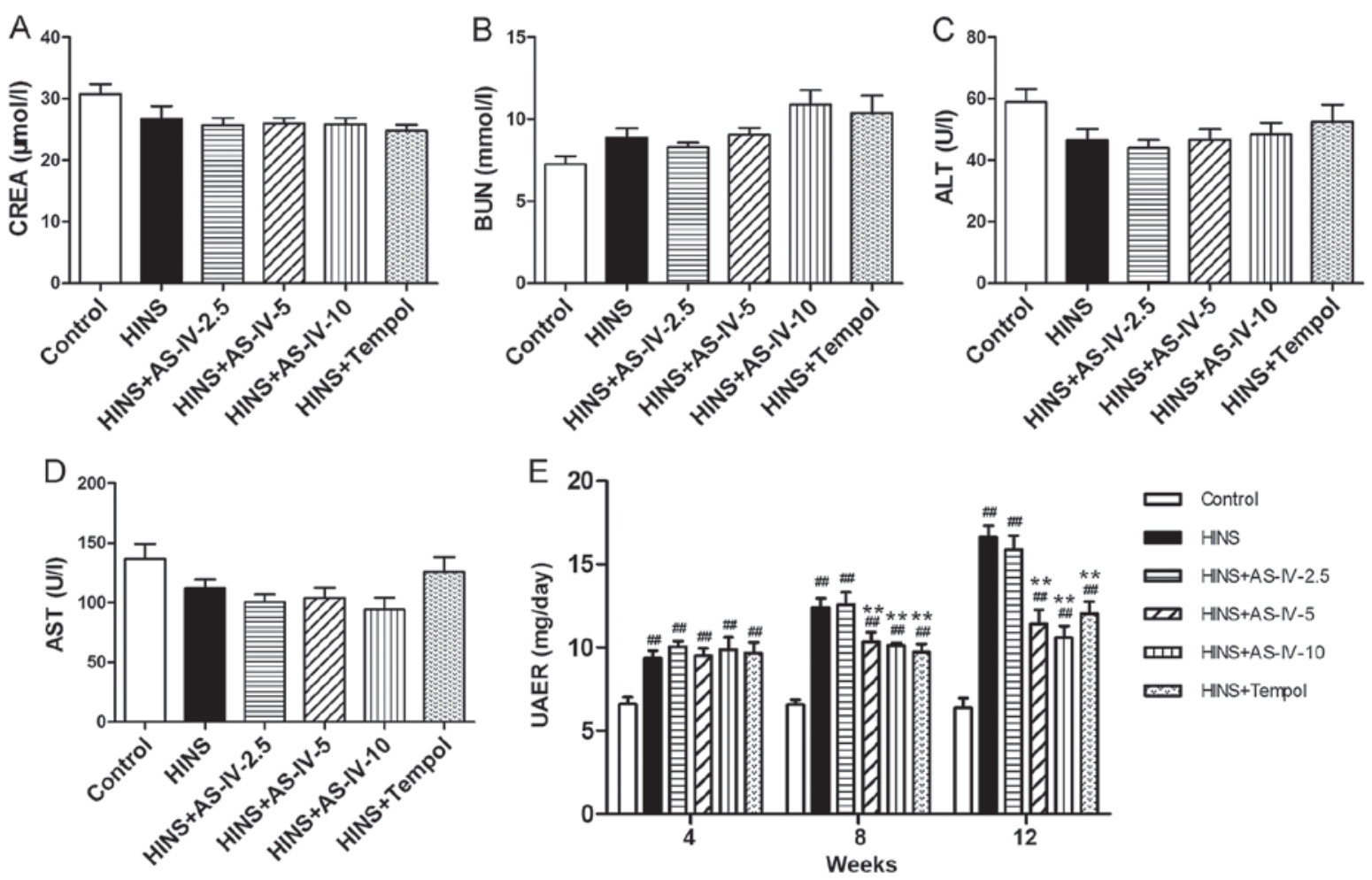

Figure 2. Effects of AS-IV on CREA, BUN, ALT, AST and UAER in iatrogenic hyperinsulinemic diabetic rats. Shown are the levels of (A) CREA, (B) BUN, (C) ALT and (D) AST of all the groups after 12 weeks. Different doses of AS-IV used in these experiments did not lead to renal or liver dysfunction. (E) Effects of AS-IV on albuminuria in diabetic rats after 4, 8 and 12 weeks. AS-IV ameliorated albuminuria of the iatrogenic hyperinsulinemic diabetic rats. Results are expressed as the means \pm standard deviation ( $\mathrm{n}=8$ /each group). Refer to the Materials and methods section for etails of the assignment of the groups ${ }^{\# \#} \mathrm{P}<0.01$ vs. control; ${ }^{* *} \mathrm{P}<0.01$ vs. HINS. AS-IV, astragaloside IV; BUN, blood urea nitrogen; ALT, alanine transaminase; AST, aspartate aminotransferase; HINS, hyperinsulinemic; UAER, urinary albumin excretion rate.
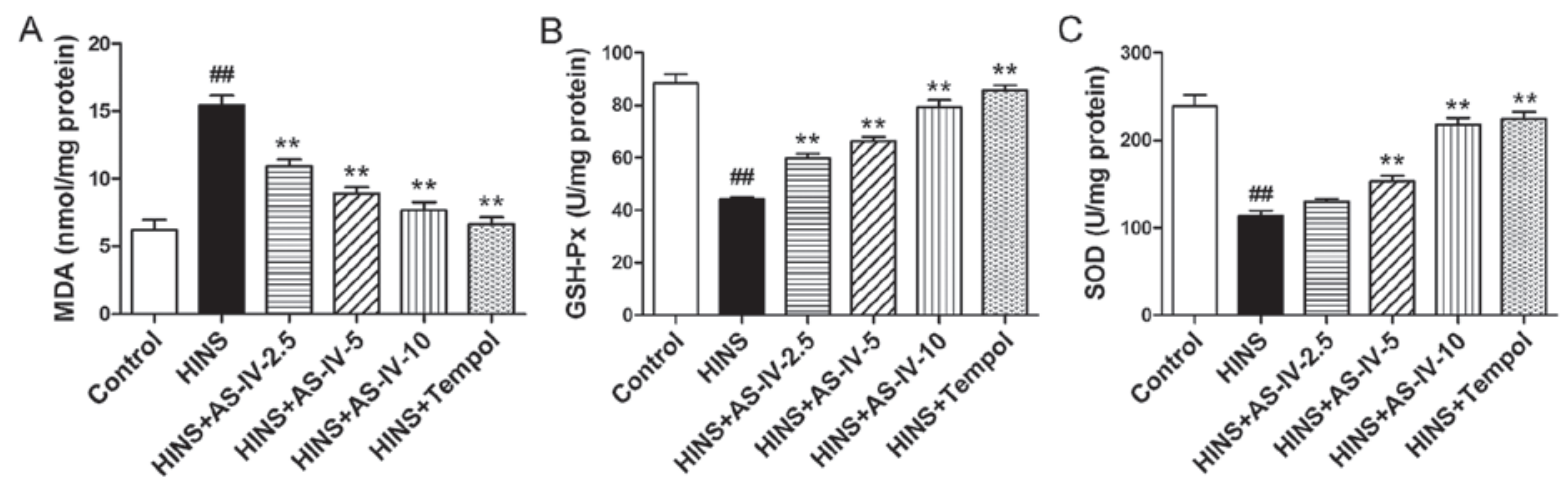

Figure 3. Effects of AS-IV on MDA, GSH-Px and SOD in iatrogenic hyperinsulinemic diabetic rats. Shown are the levels of (A) MDA, (B) GSH-Px and (C) SOD in the renal cortex of all groups after 12 weeks. AS-IV significantly reduced the elevation of renal tissue levels of MDA, and increased the elevation of the tissue levels of GSH-Px and SOD, effects that were evident at a dose as low as $2.5 \mathrm{mg} / \mathrm{kg} / \mathrm{day}$ for MDA and GSH-Px, but not for SOD (the increase for SOD was observed at a dose of AS -IV as low as $5 \mathrm{mg} / \mathrm{kg} / \mathrm{day}$ ). Results are expressed as the means $\pm \mathrm{SD}$ ( $\mathrm{n}=8 /$ each group). Refer to the Materials and methods section for details of the assignment of the groups. ${ }^{\# \#} \mathrm{P}<0.01$ vs. control; ${ }^{* *} \mathrm{P}<0.01$ vs. HINS. AS-IV, astragaloside IV; MDA, malondialdehyde; GSH-Px, glutathione peroxidase; SOD, superoxide dismutase; HINS, hyperinsulinemic.

following the successful modeling of iatrogenic hyperinsulinemia, the HINS group exhibited modest levels of mesangial cell proliferation compared with the normal control rats (Fig. 5A and B). However, AS-IV and Tempol treatment was able to prevent mesangial cell proliferation to a significant degree when compared with the HINS group. This trend was evident at a dose as low as $5 \mathrm{mg} / \mathrm{kg} / \mathrm{day}$. Fig. 5A and B show representative histopathological changes in the kidney, with $\mathrm{H} \& \mathrm{E}$ and PAS staining in each group at week 12 following AS-IV treatment. The HINS group revealed a modest level of proliferation of mesangial cells, whereas almost no changes were observed in the control, AS-IV-5, AS-IV-10 and Tempol groups.

AS-IV alleviates basement membrane thickening and podocyte foot process effacement of kidneys in iatrogenic hyperinsulinemic rats. As shown in Fig. 6, the observation of basement membrane and podocyte ultrastructure by electron 

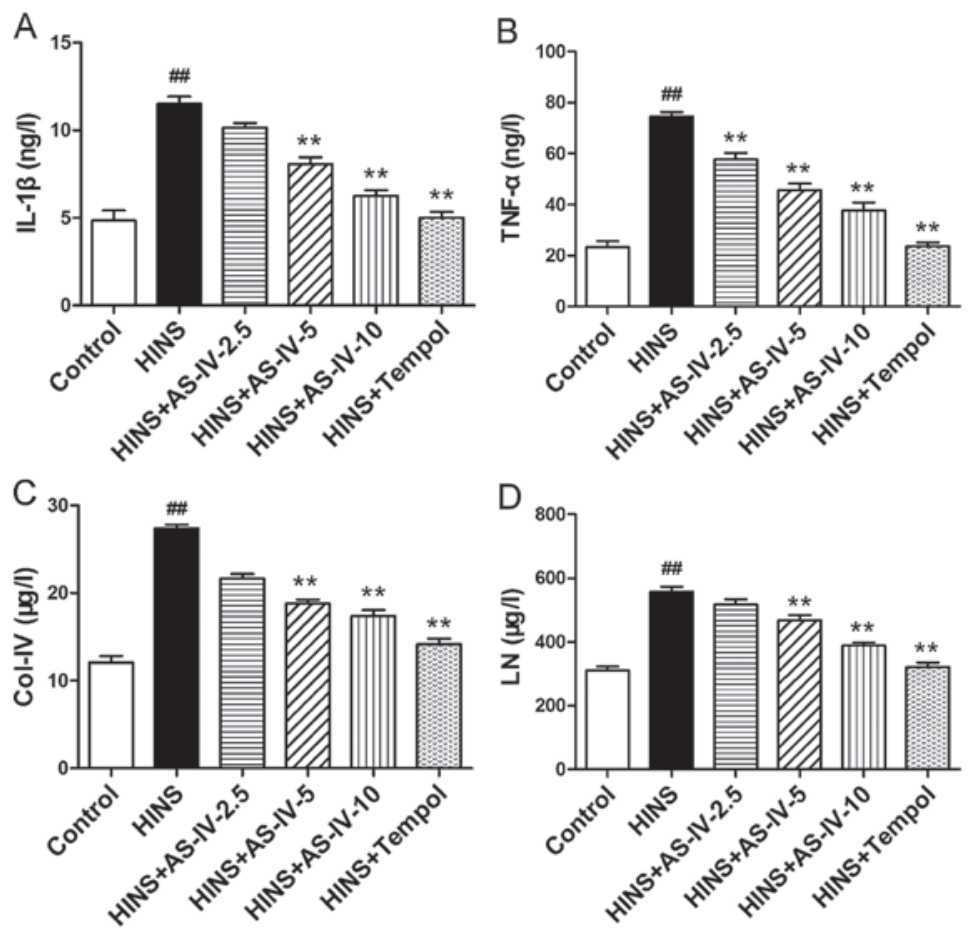

Figure 4. Effects of AS-IV on IL-1 $\beta$, TNF- $\alpha$, Col-IV and LN in iatrogenic hyperinsulinemic diabetic rats. Shown are the renal cortex levels of (A) IL-1 $\beta$, (B) TNF- $\alpha$, (C) Col-IV and (D) LN of all groups after 12 weeks. AS-IV significantly reduced the renal tissue levels of IL-1 $\beta$, TNF- $\alpha$, Col-IV and LN, which was evident at a dose as low as $2.5 \mathrm{mg} / \mathrm{kg} / \mathrm{day}(\mathrm{TNF}-\alpha)$ and $5 \mathrm{mg} / \mathrm{kg} / \mathrm{day}$ (IL-1 $\beta, \mathrm{Col}-\mathrm{IV}$ and LN). Results are expressed as the means \pm standard deviation $(\mathrm{n}=8 / \mathrm{each}$ group). Refer to the Materials and methods section for details of the assignment of the groups. ${ }^{\# t} \mathrm{P}<0.01$ vs. control; ${ }^{* *} \mathrm{P}<0.01$ vs. HINS. AS -IV, astragaloside IV; HINS, hyperinsulinemic; IL-1 $\beta$, interleukin- $1 \beta$; TNF- $\alpha$, tumor necrosis factor- $\alpha$; Col-IV, type IV collagen; LN, laminin.
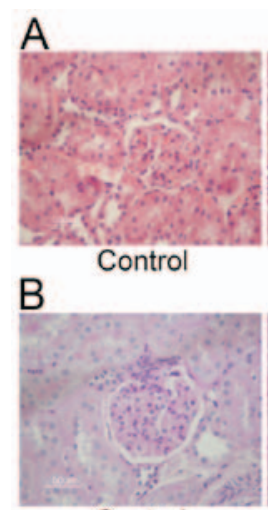

Control

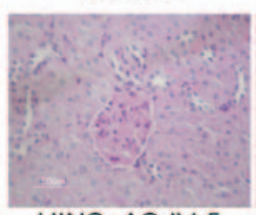

HINS+AS-IV-5

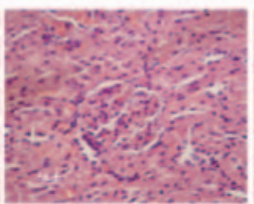

HINS

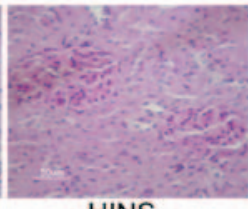

HINS

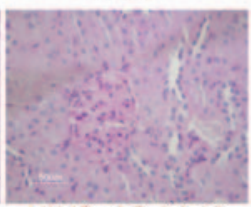

HINS+AS-IV-10

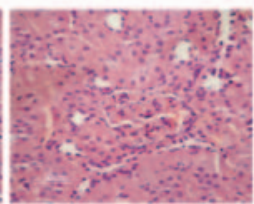

HINS+AS-IV-2.5

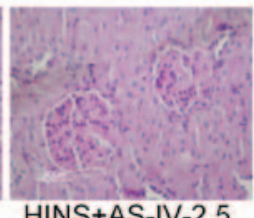

HINS+AS-IV-2.5

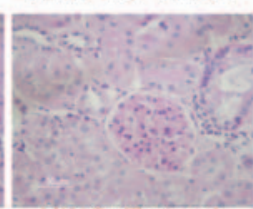

HINS+Tempol

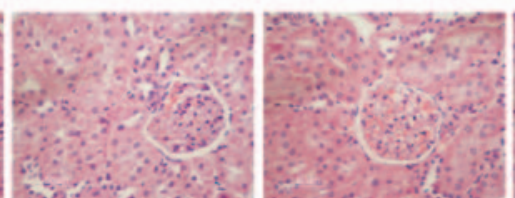

HINS+AS-IV-5

C

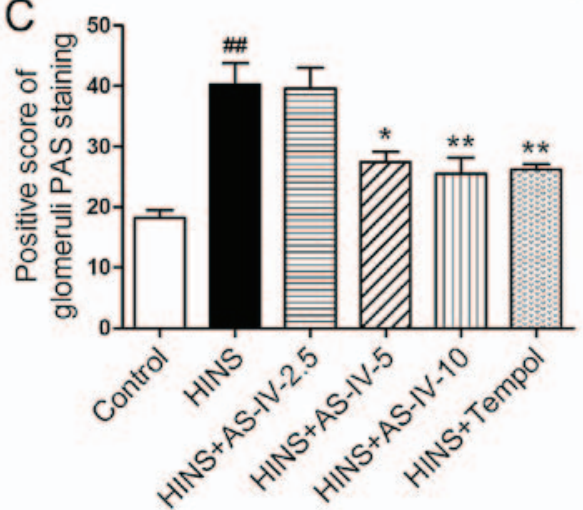

Figure 5. Representative histological changes in the kidneys of each group of rats. (A) The histological samples were stained with (A) hematoxylin and eosin or (B) with PAS. Each bar indicates $50 \mu \mathrm{m}$. (C) Positive score of glomerular PAS staining is indicated. Treatment with AS-IV for 12 weeks prevented the mesangial cell proliferation that was observed in the iatrogenic hyperinsulinemic diabetic rats, and these effects were evident at a dose of AS-IV as low as $5 \mathrm{mg} / \mathrm{kg} /$ day. Results are expressed as the means $\pm \mathrm{SD}$ (n=8/each group). Refer to the Materials and methods section for details of the assignment of the groups. ${ }^{\# \#} \mathrm{P}<0.01$ vs. control; " $\mathrm{P}<0.05$ vs. HINS; ${ }^{* *} \mathrm{P}<0.01$ vs. HINS. PAS, periodic acid Schiff; AS -IV, astragaloside IV; HINS, hyperinsulinemic.

microscopy revealed clear basement membrane thickening and minor podocyte foot process effacement in the HINS group, whereas the hyperinsulinemic rats treated with
AS-IV revealed a marked decrease in podocyte foot process effacement and basement membrane thickening, which was evident at a dose as low as $5 \mathrm{mg} / \mathrm{kg} /$ day. 


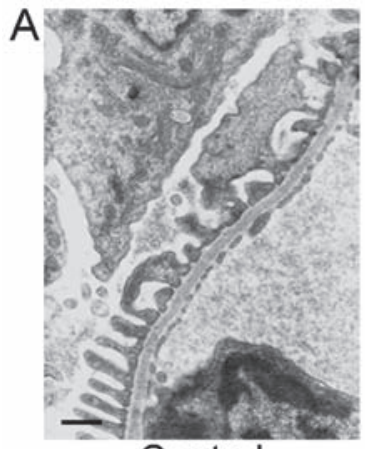

Control

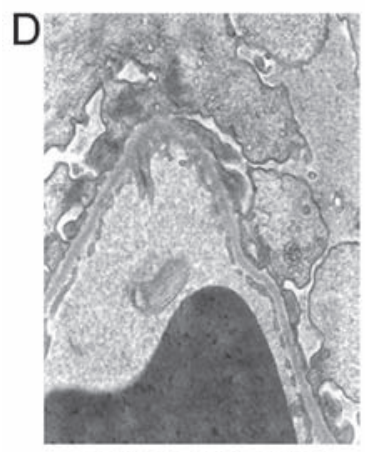

HINS+AS-IV-5

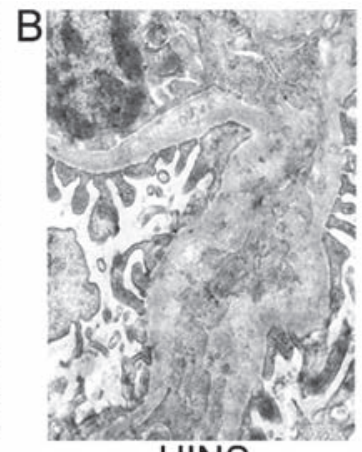

HINS

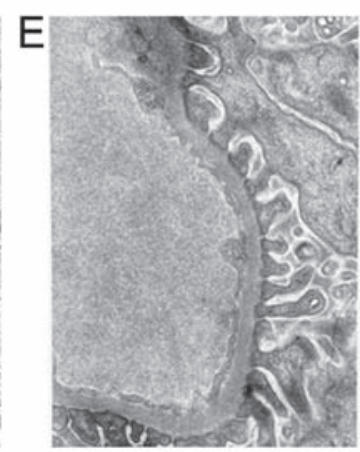

HINS+AS-IV-10
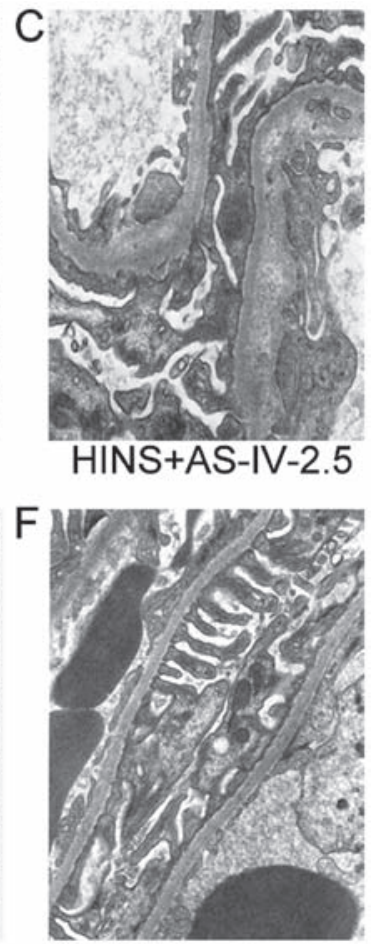

HINS+Tempol

Figure 6. Representative electron photomicrographs illustrating the changes in kidneys of each group of rats. AS-IV treatment led to a marked decrease in podocyte foot process effacement and basement membrane thickening, which was evident at a dose of AS -IV as low as 5 mg/kg/day. Refer to the Materials and methods section for details of the assignment of the groups. The bars indicate $500 \mathrm{~nm}$. AS -IV, astragaloside IV; HINS, hyperinsulinemic.

AS-IV suppresses the activation of Nox4 and ERK1/2 induced by iatrogenic hyperinsulinemia, and upregulates the expression of TRPC6 in the kidney. Having shown that AS-IV exerted antioxidative and anti-inflammatory effects, subsequently the underlying mechanism was investigated. In the kidney, particularly in mesangial cells, Nox4 is the predominant source of ROS. The ERK pathway is an important signalling pathway with regard to the proliferation of, and ECM accumulation in, renal tissue. The role of the NADPH oxidase/ERK1/2 pathway was therefore investigated. The protein expression levels of Nox4, ERK1/2, p-ERK1/2 and TRPC6 were examined by western blotting (Fig. 7A), and the results were analyzed semi-quantitatively. The HINS group exhibited elevated levels of Nox4 and phosphorylated ERK1/2 in the kidneys compared with the normal control group $(\mathrm{P}<0.01)$ (Fig. 7B). Treatment with AS-IV, however, significantly inhibited Nox4 expression and phosphorylation of ERK1/2 in the kidneys from the iatrogenic hyperinsulinemic model rats (Fig. 7B and C), which was evident at a dose as low as 2.5 and $5 \mathrm{mg} / \mathrm{kg} /$ day, respectively. TRPC6 is known as a $\mathrm{Ca}^{2+}$-conductive cation channel that regulates the contractile function of mesangial cells. Therefore, in the present study, the effects of AS-IV on the expression level of TRPC6 protein in the rat model of iatrogenic hyperinsulinemia were examined. We had investigated the expression of NADPH oxidase and TRPC6 preliminarily, trying to find the relationship between them. The results presented the relationship between them preliminarily in Fig. 7, and we are implementing cell experiments to confirm that. As illustrated in Fig. 7D, compared with the normal control group, the HINS group exhibited lower levels of TRPC6 protein $(\mathrm{P}<0.01)$. Treatment with AS-IV significantly enhanced expression of the TRPC6 protein in the kidneys, which was evident at a dose as low as $2.5 \mathrm{mg} / \mathrm{kg} / \mathrm{day}$. This dose coincided with that causing inhibition of the Nox4 expression.

\section{Discussion}

The results presented in the current study support the hypothesis that AS-IV prevents iatrogenic hyperinsulinemia due to kidney injury in STZ-induced diabetic rats through the suppression of oxidative stress, inhibiting the production of pro-inflammatory factors. This leads to a prevention of mesangial cell proliferation, basement membrane thickening and podocyte foot process effacement, thereby ameliorating albuminuria.

DN is an important complication associated with diabetes mellitus, and has become a common cause of end-stage renal failure among patients undergoing chronic hemodialysis therapy. Controlling the blood glucose level is the first issue of concern. An insufficient control may lead to the rapid onset of DN. However, patients with diabetes mellitus are able to achieve good blood glucose control by using insulin, and yet, ultimately, the onset of DN cannot be avoided. This demonstrates that, despite our ability to tightly control the blood glucose level, development of DN is not determined by a single factor associated with blood glucose. Certain researchers have gone so far as to propose that intensive insulin treatment does not protect renal function (25). Though not all patients with diabetes mellitus have hyperinsulinemia, patients with type 1 diabetes (3), and type 2 diabetic patients who are using insulin $(26,27)$, have been observed to have iatrogenic hyperinsulinemia. With respect to the establishment of a rat model, the clinical processes of patients with diabetes mellitus with iatrogenic hyperinsulinemia were 

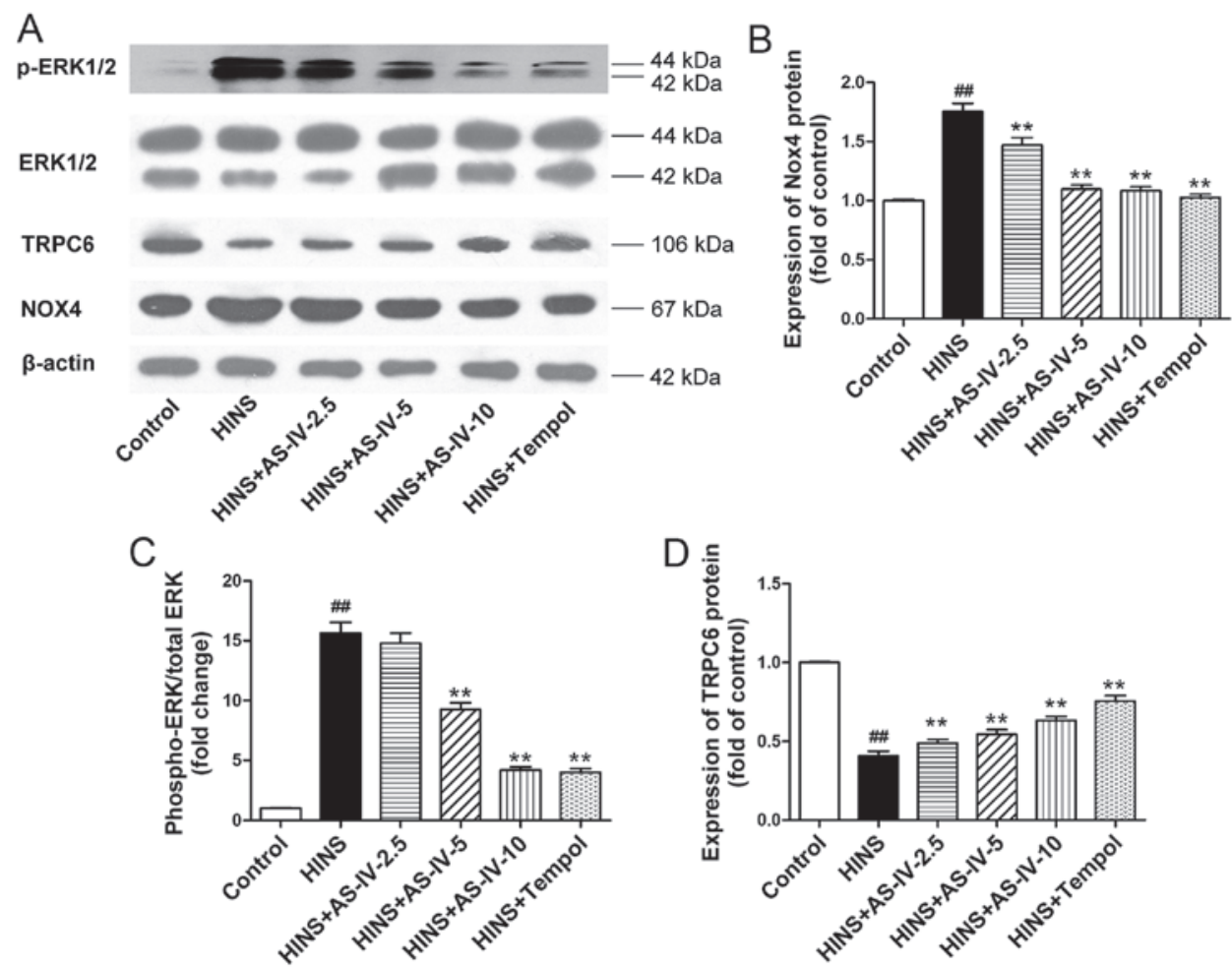

Figure 7. AS-IV regulates the protein expression of Nox4, ERK1/2, p-ERK1/2 and TRPC6 in iatrogenic hyperinsulinemic diabetic rats. (A) The protein expression of Nox4, ERK1/2, p-ERK1/2, TRPC6 and $\beta$-actin was examined by western blotting, and the results of semi-quantitative analysis of the data for (B) Nox4, (C) p-ERK1/2/ERK1/2 and (D) TRPC6 are also shown. $\beta$-actin was used as a loading control. AS-IV significantly inhibited the expression of Nox4 and phosphorylation of ERK1/2 in the kidneys from the iatrogenic hyperinsulinemic model rats, an effect that was evident at a dose of AS -IV as low as 2.5 and $5 \mathrm{mg} / \mathrm{kg} / \mathrm{day}$ for Nox4 and p-ERK1/2/ERK1/2, respectively. AS-IV significantly enhanced expression of the TRPC6 protein in the kidneys, which was evident at a dose as low as $2.5 \mathrm{mg} / \mathrm{kg} / \mathrm{day}$. Results are expressed as the means \pm standard deviation ( $\mathrm{n}=8 /$ each group). The experiments were performed in triplicate. ${ }^{\# \prime} \mathrm{P}<0.01$ vs. control; ${ }^{* *} \mathrm{P}<0.01$ vs. HINS. AS-IV, astragaloside IV; HINS, hyperinsulinemic; Nox4, NADPH oxidase 4; ERK1/2, extracellular signal-regulated kinase 1/2; p -, phosphorylated; TRPC6, transient receptor potential cation channel 6 .

simulated in the present study. First, the model of diabetic rats was established. Secondly, iatrogenic hyperinsulinemic diabetic rats were induced by subcutaneous injection of insulin. The main purpose in the establishment of this model was to obtain effective control of the blood glucose level, in order to study the impact of iatrogenic hyperinsulinemia on the kidney. As described in the Results section, AS-IV did not change blood glucose or insulin levels. This suggested that AS-IV was not able to exert any impact on the blood glucose level: Only insulin itself could. More importantly, our rat model simulated intensive insulin treatment. The goal of intensive glycemic control is to regulate blood glucose and $\mathrm{HbA}_{1 \mathrm{c}}$ levels within the normal range (28). According to a preliminary experiment, Levemir at a dose of $6 \mathrm{U} /$ day was able to control the blood glucose level well. Therefore, the hyperinsulinemic rats had almost normal blood glucose and $\mathrm{HbA}_{1 \mathrm{c}}$ levels compared with normal control rats. The difference between hyperinsulinemic rats and normal control rats was attributable solely to their insulin levels. In order to minimize the interfering factors, a high-sugar and high-fat manufacturing model was not employed. Furthermore, nephrotoxicity is not likely to have resulted from the use of STZ, since the dose of STZ $(55 \mathrm{mg} / \mathrm{kg})$ used to induce diabetes has been reported to have minimal kidney toxicity in experimental animals (29). Iatrogenic or exogenous hyperinsulinemia is an unavoidable consequence of the direct administration of insulin into the general circulation (6).
Therefore, in our model, the generation of iatrogenic hyperinsulinemia that was observed was expected.

Radix Astragali, the dried root of Astragalus membranaceus (Fisch.) Bunge, has long been used in traditional Chinese medicine for the treatment of diabetes. AS-IV is a novel saponin extracted from Radix Astragali, which prevents damage to human mesangial cells and umbilical vein endothelial cells by suppressing oxidative stress, inflammation and cell proliferation, as demonstrated in previous studies by our group $(7,30)$. High concentrations of insulin are able to promote cell proliferation, ECM expansion and oxidative stress in vitro. This therefore suggested the use of AS-IV in vivo to intervene with renal damage caused by iatrogenic hyperinsulinemia, as long as it proved to be effective.

The most important clinical manifestation of DN is albuminuria (31). In the current study, the amounts of albumin in the urine of diabetic rats were low. Insulin has a therapeutic effect on diabetes mellitus. If appreciable levels of albuminuria were to have been observed, it was possible that our study could have been implemented for a longer period of time. Though the overall level of kidney damage was minor, rats were observed during the very early period of DN. Direct observation of histological sections stained with H\&E and PAS revealed basement membrane thickening in the HINS group. Subsequently, electron microscopy (magnification, $\mathrm{x} 15,000$ ) was used to observe basement membrane thickening and podocyte foot process effacement. AS-IV was able to prevent 
these pathological changes, and these were the most intuitive results obtained in this study, providing important evidence for renal function protection mediated by AS-IV, results which are consistent with other studies $(21,32)$.

Subsequently, the mechanism underlying the changes described above was sought after. Oxidative stress and inflammation are the two predominant factors of DN (33). First, in a high-insulin environment, levels of oxidative stress may be increased (34), and endogenous hyperinsulinemia can exacerbate oxidative stress (35). Piwkowska et al (36) demonstrated that high insulin levels increase the glomerular barrier albumin permeability via a protein kinase G type I (PKGI)-dependent mechanism involving $\mathrm{NAD}(\mathrm{P}) \mathrm{H}$-dependent generation of superoxide anion in podocytes. Markers of oxidative stress, including ROS and reduced levels of antioxidants, have been identified in renal tissues in human and experimental models of diabetes (37). The excessive production of ROS is an important mechanism underlying the pathogenesis of diabetes-associated DN. The level of MDA provides a good index of intensified oxidative stress in tissues exhibiting enhanced peroxidation processes (38). SOD and GSH-Px constitute the principal components of the cohort of cellular antioxidants, and their deficiencies can cause oxidative stress. The SOD enzyme system is a primary determinant of superoxide removal (39). Changes in the levels of the above indicators were observed in the present study, implying that renal oxidative stress was present. The antioxidant effect mediated by AS-IV was evident in the iatrogenic hyperinsulinemic rats. Secondly, inflammation exerts a pivotal role in the pathogenesis of DN. Hyperinsulinemia may increase inflammation and pro-inflammatory cytokine release (40). Certain pro-inflammatory cytokines, including IL-1 and TNF- $\alpha$, have been reported to participate in the pathogenesis of DN (41). ROS generation was also noted to be associated with increased levels of TNF- $\alpha$ and increased glomerular lesions in experimental diabetic rats (42), suggesting a role for free radical generation in association with TNF- $\alpha$ in the induction and progression of diabetes-associated renal injury. Taken together, the results in the present study suggested that renal inflammation was present. The anti-inflammatory effect of AS-IV was evident in the iatrogenic hyperinsulinemic rats, results which are consistent with the study of Gui et al (32).

Col-IV and LN are important components of the basement membrane, and important indicators of ECM accumulation. Excessive deposition of Col-IV is an established feature associated with diabetic glomerulopathy (43), whereas aberrant expression of $\mathrm{LN}$ is a critical pathological characteristic of DN (44).

Oxidative stress is able to promote anti-glomerular basement membrane glomerulonephritis in renin-angiotensin system activation in the kidney (45), and LN production in renal mesangial cells (46). However, it was reported that inflammation could promote $\mathrm{LN}$ expression (47). It was also reported that high insulin levels are able to promote ECM synthesis in vitro (48). The ERK pathway is an important signalling pathway involved with the proliferation of renal tissue (49) and with the ECM synthesis that occurs during scar formation (50). Hyperinsulinemia is able to activate the ERK pathway (51). In the kidney, oxidative stress can activate the ERK pathway (52), and inflammation may also activate the ERK pathway in the kidney (53). Therefore, it was hypothesized that, in the iatrogenic hyperinsulinemic environment, oxidative stress and inflammation in kidney tissues were enhanced, and the two processes served to co-activate the ERK pathway, which resulted in cell proliferation and increased ECM deposition. The possible mechanisms in operation in the hyperinsulinemic environment of mesangial cells and podocytes are as follows: First, from our previous study, high insulin levels induce an increase in Nox4-sourced ROS production, and ROS further promote mesangial cell proliferation and increases in ECM deposition (54). Secondly, as for the podocytes, it was reported that insulin increases the glomerular filtration barrier permeability through PKGI $\alpha$-dependent mobilization of large conductance calcium-activated potassium (BKCa) channels (55). Thus, effacement of the podocyte foot processes occurs, and albuminuria appears. Based on the results in the present study, AS-IV exerts clear anti-inflammatory effects and inhibits oxidative stress, thereby inhibiting mesangial cell proliferation and basement membrane thickening. However, the observed effects of mesangial cell proliferation and basement membrane thickening levels were modest. This may be due to the relatively short experimental period. In establishing the experimental design, it was a concern that extended s.c. injections of insulin could induce exogenous insulin resistance, or even subcutaneous insulin resistance.

TRPC6 is an important protein in the maintenance of normal renal function (56). It protects against renal ischemia-reperfusion injury (57). In the present study, the expression of TRPC6 was reduced in the iatrogenic hyperinsulinemic environment. This reduction may have been associated with the oxidative stress environment produced by iatrogenic hyperinsulinemia, and this result was consistent with our previous study (24). The possible reason has yet to be elucidated, although the mechanism may be that TRPC6 protein is decreased by ROS (58). The possible mechanism will be explored further in our subsequent studies. AS-IV is able to reduce the degree of the TRPC6 protein decreases in iatrogenic hyperinsulinemia by upregulating the expression of TRPC6.

The study drug selected for the present study was a traditional Chinese medicine monomer, and its pharmacological mechanism has yet to be fully elucidated. Previous studies have shown that it has anti-inflammatory effects and inhibits oxidative stress. It has been reported that the NADPH oxidase inhibitor, apocynin, protects the kidney against DN in Otsuka Long Evans Tokushima Fatty (OLETF) rats (14). As observed previously for anti-oxidative stress drugs, Tempol exerts anti-inflammatory and antioxidant effects, and it has also been reported to have kidney-protective effects (24). Tempol was therefore selected as a positive control drug.

In conclusion, AS-IV prevents kidney injury in iatrogenic hyperinsulinemic diabetic rats by suppressing oxidative stress, inhibiting IL-1 $\beta$ and TNF- $\alpha$ overproduction, downregulating ERK1/2 activation, and upregulating TRPC6 expression in the field of DN. The findings in the present study should provide a method for delaying the occurrence of DN under intensive insulin treatment.

\section{Acknowledgements}

This study was supported by grants from the National Natural Science Foundation of China (no. 81173624), the Nature 
Science Foundation of Anhui Province (no. 11040606M201), the College Natural Science Research Project of Anhui province (no. KJ2016SD35), and the International Scientific and Technological Cooperative Project of Anhui province (no. 1230603007). The authors would like to thank Dr Li Gui and Dake Huang from the Synthetic Laboratory of Anhui Medical University for their helpful technical assistance.

\section{References}

1. American Diabetes Association: Standards of medical care in diabetes - 2011. Diabetes Care 34 (Suppl 1): S11-S61, 2011.

2. Choudhury D, Tuncel M and Levi M: Diabetic nephropathy - - a multifaceted target of new therapies. Discov Med 10: 406-415, 2010.

3. Wang MY, Yu X, Lee Y, McCorkle SK, Clark GO, Strowig S, Unger RH and Raskin P: Iatrogenic hyperinsulinemia in type 1 diabetes: Its effect on atherogenic risk markers. J Diabetes Complications 27: 70-74, 2013.

4. Mariappan MM, DeSilva K, Sorice GP, Muscogiuri G, Jimenez F, Ahuja S, Barnes JL, Choudhury GG, Musi N, DeFronzo R, et al: Combined acute hyperglycemic and hyperinsulinemic clamp induced profibrotic and proinflammatory responses in the kidney. Am J Physiol Cell Physiol 306: C202-C211, 2014.

5. Ngubane PS, Hadebe SI, Serumula MR and Musabayane CT: The effects of transdermal insulin treatment of streptozotocin-induced diabetic rats on kidney function and renal expression of glucose transporters. Ren Fail 37: 151-159, 2015.

6. Draznin B, Miles P, Kruszynska Y, Olefsky J, Friedman J, Golovchenko I, Stjernholm R, Wall K, Reitman M, Accili D, et al: Effects of insulin on prenylation as a mechanism of potentially detrimental influence of hyperinsulinemia. Endocrinology 141: 1310-1316, 2000.

7. Sun L, Li W, Li W, Xiong L, Li G and Ma R: Astragaloside IV prevents damage to human mesangial cells through the inhibition of the NADPH oxidase/ROS/Akt/NF- $\kappa$ B pathway under high glucose conditions. Int J Mol Med 34: 167-176, 2014.

8. Kim EY, Anderson M and Dryer SE: Insulin increases surface expression of TRPC6 channels in podocytes: Role of NADPH oxidases and reactive oxygen species. Am J Physiol Renal Physiol 302: F298-F307, 2012.

9. Hong OK, Lee SH, Rhee M, Ko SH, Cho JH, Choi YH, Song KH, Son HY and Yoon KH: Hyperglycemia and hyperinsulinemia have additive effects on activation and proliferation of pancreatic stellate cells: Possible explanation of islet-specific fibrosis in type 2 diabetes mellitus. J Cell Biochem 101: 665-675, 2007.

10. Mariappan MM, Feliers D, Mummidi S, Choudhury GG and Kasinath BS: High glucose, high insulin, and their combination rapidly induce laminin-betal synthesis by regulation of mRNA translation in renal epithelial cells. Diabetes 56: 476-485, 2007.

11. Bondi CD, Manickam N, Lee DY, Block K, Gorin Y, Abboud HE and Barnes JL: NAD(P)H oxidase mediates TGF-betal-induced activation of kidney myofibroblasts. J Am Soc Nephrol 21: 93-102, 2010.

12. Asaba K, Tojo A, Onozato ML, Goto A, Quinn MT, Fujita T and Wilcox CS: Effects of NADPH oxidase inhibitor in diabetic nephropathy. Kidney Int 67: 1890-1898, 2005.

13. Gorin Y, Block K, Hernandez J, Bhandari B, Wagner B, Barnes JL and Abboud HE: Nox4 NAD(P)H oxidase mediates hypertrophy and fibronectin expression in the diabetic kidney. J Biol Chem 280: 39616-39626, 2005.

14. Nam SM, Lee MY, Koh JH, Park JH, Shin JY, Shin YG, Koh SB, Lee EY and Chung CH: Effects of NADPH oxidase inhibitor on diabetic nephropathy in OLETF rats: The role of reducing oxidative stress in its protective property. Diabetes Res Clin Pract 83: 176-182, 2009.

15. Mora $\mathrm{C}$ and Navarro JF: Inflammation and pathogenesis of diabetic nephropathy. Metabolism 53: 265-266, author reply 266-267, 2004

16. Huang Y, Zeng J, Chen G, Xie X, Guo W and Tian W: Periodontitis contributes to adipose tissue inflammation through the NF-kappaB, JNK and ERK pathways to promote insulin resistance in a rat model. Microbes Infect 18: 804-812, 2016.
17. Smyth JT, Hwang SY, Tomita T, DeHaven WI, Mercer JC and Putney JW: Activation and regulation of store-operated calcium entry. J Cell Mol Med 14: 2337-2349, 2010.

18. Nilius B and Owsianik G: The transient receptor potential family of ion channels. Genome Biol 12: 218, 2011.

19. Ma R, Du J, Sours S and Ding M: Store-operated $\mathrm{Ca}^{2+}$ channel in renal microcirculation and glomeruli. Exp Biol Med (Maywood) 231: 145-153, 2006.

20. Ren S, Zhang H, Mu Y, Sun M and Liu P: Pharmacological effects of astragaloside IV: A literature review. J Tradit Chin Med 33: 413-416, 2013.

21. Gui D, Huang J, Liu W, Guo Y, Xiao W and Wang N: Astragaloside IV prevents acute kidney injury in two rodent models by inhibiting oxidative stress and apoptosis pathways. Apoptosis 18: 409-422, 2013.

22. Danda RS, Habiba NM, Rincon-Choles H, Bhandari BK, Barnes JL, Abboud HE and Pergola PE: Kidney involvement in a nongenetic rat model of type 2 diabetes. Kidney Int 68: 2562-2571, 2005

23. Banse HE, Frank N, Kwong GP and McFarlane D: Relationship of oxidative stress in skeletal muscle with obesity and obesity-associated hyperinsulinemia in horses. Can J Vet Res 79: 329-338, 2015.

24. Luan J,Li W,Han J,Zhang W, Gong H and Ma R: Renal protection of in vivo administration of tempol in streptozotocin-induced diabetic rats. J Pharmacol Sci 119: 167-176, 2012.

25. Chowdhury TA, O'Toole S and Yaqoob MM: Managing blood glucose levels in patients with diabetes and renal impairment. Br J Hosp Med (Lond) 77: C10-C13, 2016.

26. Velussi M, Cernigoi AM, De Monte A, Dapas F, Caffau C and Zilli M: Long-term (12 months) treatment with an anti-oxidant drug (silymarin) is effective on hyperinsulinemia, exogenous insulin need and malondialdehyde levels in cirrhotic diabetic patients. J Hepatol 26: 871-879, 1997.

27. Müssig K, Staiger H, Kantartzis K, Fritsche A, Kanz L and Häring HU: Type 2 diabetes mellitus and risk of malignancy: Is there a strategy to identify a subphenotype of patients with increased susceptibility to endogenous and exogenous hyperinsulinism? Diabet Med 28: 276-286, 2011.

28. Gerstein HC, Bosch J, Dagenais GR, Díaz R, Jung H, Maggioni AP, Pogue J, Probstfield J, Ramachandran A, Riddle MC, et al; ORIGIN Trial Investigators: Basal insulin and cardiovascular and other outcomes in dysglycemia. N Engl J Med 367: 319-328, 2012.

29. Koulmanda M, Qipo A, Chebrolu S, O'Neil J, Auchincloss H and Smith RN: The effect of low versus high dose of streptozotocin in cynomolgus monkeys (Macaca fascilularis). Am J Transplant 3: 267-272, 2003

30. Ma Y, Li W, Yin Y and Li W: AST IV inhibits $\mathrm{H}_{2} \mathrm{O}_{2}$-induced human umbilical vein endothelial cell apoptosis by suppressing Nox4 expression through the TGF- $\beta 1 /$ Smad2 pathway. Int J Mol Med 35: 1667-1674, 2015

31. Gluhovschi C, Gluhovschi G, Petrica L, Timar R, Velciov S, Ionita I, Kaycsa A and Timar B: Urinary biomarkers in the assessment of early diabetic nephropathy. J Diabetes Res 2016: $4626125,2016$.

32. Gui D, Huang J, Guo Y, Chen J, Chen Y, Xiao W, Liu X and Wang N: Astragaloside IV ameliorates renal injury in streptozotocin-induced diabetic rats through inhibiting NF- $\mathrm{KB}$-mediated inflammatory genes expression. Cytokine 61: 970-977, 2013.

33. Voroneanu L, Nistor I, Dumea R, Apetrii M and Covic A: Silymarin in Type 2 Diabetes Mellitus: A systematic review and meta-analysis of randomized controlled trials. J Diabetes Res 2016: 5147468, 2016.

34. Abhijit S, Bhaskaran R, Narayanasamy A, Chakroborty A, Manickam N, Dixit M, Mohan V and Balasubramanyam M: Hyperinsulinemia-induced vascular smooth muscle cell (VSMC) migration and proliferation is mediated by converging mechanisms of mitochondrial dysfunction and oxidative stress. Mol Cell Biochem 373: 95-105, 2013.

35. Xu L and Badr MZ: Enhanced potential for oxidative stress in hyperinsulinemic rats: imbalance between hepatic peroxisomal hydrogen peroxide production and decomposition due to hyperinsulinemia. Horm Metab Res 31: 278-282, 1999.

36. Piwkowska A, Rogacka D, Kasztan M, Angielski S and Jankowski M: Insulin increases glomerular filtration barrier permeability through dimerization of protein kinase $\mathrm{G}$ type I $\alpha$ subunits. Biochim Biophys Acta 1832: 791-804, 2013.

37. Lee HB, Yu MR, Yang Y, Jiang Z and Ha H: Reactive oxygen species-regulated signaling pathways in diabetic nephropathy. J Am Soc Nephrol 14 (Suppl 3): S241-S245, 2003. 
38. Kedziora-Kornatowska K, Szram S, Kornatowski T, Szadujkis-Szadurski L, Kedziora J and Bartosz G: The effect of verapamil on the antioxidant defence system in diabetic kidney. Clin Chim Acta 322: 105-112, 2002.

39. Fridovich I: Superoxide radical and superoxide dismutases. Annu Rev Biochem 64: 97-112, 1995.

40. Liu S, Zhang Q, Chen C, Ge D, Qu Y, Chen R, Fan YM, Li N, Tang WW, Zhang W, et al: Hyperinsulinemia enhances interleukin-17-induced inflammation to promote prostate cancer development in obese mice through inhibiting glycogen synthase kinase 3-mediated phosphorylation and degradation of interleukin-17 receptor. Oncotarget 7: 13651-13666, 2016.

41. Hasegawa G, Nakano K, Sawada M, Uno K, Shibayama Y, Ienaga $\mathrm{K}$ and Kondo M: Possible role of tumor necrosis factor and interleukin-1 in the development of diabetic nephropathy. Kidney Int 40: 1007-1012, 1991.

42. Xiao H, Li Y, Qi J, Wang H and Liu K: Peroxynitrite plays a key role in glomerular lesions in diabetic rats. J Nephrol 22: 800-808, 2009.

43. Pugliese G, Pricci F, Pugliese F, Mene P, Lenti L, Andreani D, Galli G, Casini A, Bianchi S, Rotella CM, et al: Mechanisms of glucose-enhanced extracellular matrix accumulation in rat glomerular mesangial cells. Diabetes 43: 478-490, 1994.

44. Setty S, Michael AA, Fish AJ, Michael Mauer S, Butkowski RJ, Virtanen I and Kim Y: Differential expression of laminin isoforms in diabetic nephropathy and other renal diseases. Mod Pathol 25: 859-868, 2012

45. Kinoshita Y, Kondo S, Urushihara M, Suga K, Matsuura S, Takamatsu M, Shimizu M, Nishiyama A, Kawachi H and Kagami S: Angiotensin II type I receptor blockade suppresses glomerular renin-angiotensin system activation, oxidative stress, and progressive glomerular injury in rat anti-glomerular basement membrane glomerulonephritis. Transl Res 158: 235-248, 2011.

46. Singh LP, Cheng DW, Kowluru R, Levi E and Jiang Y: Hexosamine induction of oxidative stress, hypertrophy and laminin expression in renal mesangial cells: Effect of the anti-oxidant alpha-lipoic acid. Cell Biochem Funct 25: 537-550, 2007.

47. Ji K and Tsirka SE: Inflammation modulates expression of laminin in the central nervous system following ischemic injury. J Neuroinflammation 9: 159, 2012.

48. Mariappan MM, Shetty M, Sataranatarajan K, Choudhury GG and Kasinath BS: Glycogen synthase kinase 3beta is a novel regulator of high glucose- and high insulin-induced extracellular matrix protein synthesis in renal proximal tubular epithelial cells. J Biol Chem 283: 30566-30575, 2008.
49. Brenner BM, Cooper ME, de Zeeuw D, Keane WF, Mitch WE, Parving HH, Remuzzi G, Snapinn SM, Zhang Z and Shahinfar S; RENAAL Study Investigators: Effects of losartan on renal and cardiovascular outcomes in patients with type 2 diabetes and nephropathy. N Engl J Med 345: 861-869, 2001.

50. Hu X, Wang H, Liu J, Fang X, Tao K, Wang Y, Li N, Shi J, Wang Y, Ji P, et al: The role of ERK and JNK signaling in connective tissue growth factor induced extracellular matrix protein production and scar formation. Arch Dermatol Res 305: 433-445, 2013.

51. Fernandez-Twinn DS, Blackmore HL, Siggens L, Giussani DA, Cross CM, Foo R and Ozanne SE: The programming of cardiac hypertrophy in the offspring by maternal obesity is associated with hyperinsulinemia, AKT, ERK, and mTOR activation. Endocrinology 153: 5961-5971, 2012.

52. He T, Guan X, Wang S, Xiao T, Yang K, Xu X, Wang J and Zhao J: Resveratrol prevents high glucose-induced epithelial-mesenchymal transition in renal tubular epithelial cells by inhibiting NADPH oxidase/ROS/ERK pathway. Mol Cell Endocrinol 402: 13-20, 2015.

53. Ma JQ, Ding J, Xiao ZH and Liu CM: Puerarin ameliorates carbon tetrachloride-induced oxidative DNA damage and inflammation in mouse kidney through ERK/Nrf2/ARE pathway. Food Chem Toxicol 71: 264-271, 2014

54. Li G, Li W, Duan W and Li W: Protective effects of As-IV on high insulin induced human mesangial cells injury and its mechanisms. Acta Univ Medicinalis Anhui 50: 1629-1633, 2015 (In Chinese).

55. Piwkowska A, Rogacka D, Audzeyenka I, Kasztan M, Angielski S and Jankowski M: Insulin increases glomerular filtration barrier permeability through PKGI $\alpha$-dependent mobilization of BKCa channels in cultured rat podocytes. Biochim Biophys Acta 1852: 1599-1609, 2015

56. Reiser J, Polu KR, Möller CC, Kenlan P, Altintas MM, Wei C, Faul C, Herbert S, Villegas I, Avila-Casado C, et al: TRPC6 is a glomerular slit diaphragm-associated channel required for normal renal function. Nat Genet 37: 739-744, 2005.

57. Shen B, He Y, Zhou S, Zhao H, Mei M and Wu X: TRPC6 may protect renal ischemia-reperfusion injury through inhibiting necroptosis of renal tubular epithelial cells. Med Sci Monit 22: 633-641, 2016.

58. Graham S, Gorin Y, Abboud HE, Ding M, Lee DY, Shi H, Ding Y and Ma R: Abundance of TRPC6 protein in glomerular mesangial cells is decreased by ROS and PKC in diabetes. Am J Physiol Cell Physiol 301: C304-C315, 2011. 